\title{
Front Matter: Volume 7579
}

, "Front Matter: Volume 7579," Proc. SPIE 7579, Laser Resonators and Beam Control XII, 757901 (22 March 2010); doi: 10.1117/12.855213

SPIE. Event: SPIE LASE, 2010, San Francisco, California, United States 


\title{
PROCEEDINGS OF SPIE
}

\section{Laser Resonators and Beam Control XII}

\author{
Alexis V. Kudryashov \\ Alan H. Paxton \\ Vladimir S. Ilchenko \\ Lutz Aschke \\ Editors
}

24-27 January 2010

San Francisco, California, United States

Sponsored and Published by

SPIE 
The papers included in this volume were part of the technical conference cited on the cover and title page. Papers were selected and subject to review by the editors and conference program committee. Some conference presentations may not be available for publication. The papers published in these proceedings reflect the work and thoughts of the authors and are published herein as submitted. The publisher is not responsible for the validity of the information or for any outcomes resulting from reliance thereon.

Please use the following format to cite material from this book:

Author(s), "Title of Paper," in Laser Resonators and Beam Control XII, edited by Alexis V. Kudryashov, Alan H. Paxton, Vladimir S. Ilchenko, Lutz Aschke, Proceedings of SPIE Vol. 7579 (SPIE, Bellingham, WA, 2010) Article CID Number.

ISSN 0277-786X

ISBN 9780819479754

Published by

SPIE

P.O. Box 10, Bellingham, Washington 98227-0010 USA

Telephone +1 3606763290 (Pacific Time) · Fax +1 3606471445

SPIE.org

Copyright (C) 2010, Society of Photo-Optical Instrumentation Engineers

Copying of material in this book for internal or personal use, or for the internal or personal use of specific clients, beyond the fair use provisions granted by the U.S. Copyright Law is authorized by SPIE subject to payment of copying fees. The Transactional Reporting Service base fee for this volume is $\$ 18.00$ per article (or portion thereof), which should be paid directly to the Copyright Clearance Center (CCC), 222 Rosewood Drive, Danvers, MA 01923. Payment may also be made electronically through CCC Online at copyright.com. Other copying for republication, resale, advertising or promotion, or any form of systematic or multiple reproduction of any material in this book is prohibited except with permission in writing from the publisher. The CCC fee code is 0277-786X/10/\$18.00.

Printed in the United States of America.

Publication of record for individual papers is online in the SPIE Digital Library.

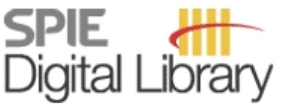

SPIEDigitalLibrary.org

Paper Numbering: Proceedings of SPIE follow an e-First publication model, with papers published first online and then in print and on CD-ROM. Papers are published as they are submitted and meet publication criteria. A unique, consistent, permanent citation identifier (CID) number is assigned to each article at the time of the first publication. Utilization of CIDs allows articles to be fully citable as soon they are published online, and connects the same identifier to all online, print, and electronic versions of the publication. SPIE uses a six-digit CID article numbering system in which:

- The first four digits correspond to the SPIE volume number.

- The last two digits indicate publication order within the volume using a Base 36 numbering system employing both numerals and letters. These two-number sets start with 00, 01, 02, 03, 04 , 05, 06, 07, 08, 09, OA, OB ... 0Z, followed by 10-1Z, 20-2Z, etc.

The CID number appears on each page of the manuscript. The complete citation is used on the first page, and an abbreviated version on subsequent pages. Numbers in the index correspond to the last two digits of the six-digit CID number. 


\section{Contents}

vii Conference Committee

\section{ADAPTIVE OPTICS}

757905 Compact fast multi-wavelength switchable single frequency laser [7579-14]

C. Moser, L. Ho, F. Havermeyer, Ondax, Inc. (United States)

757906 New paradigms for old problems: some (small) advances in laser resonator research at the CSIR (Invited Paper) [7579-05]

A. Forbes, CSIR National Laser Ctr. (South Africa) and Univ. of KwaZulu-Natal (South Africa)

\section{ADAPTIVE OPTICS AND LASER SIMULATION}

757907 Saturation characteristics of gain guiding in index-antiguided waveguides [7579-07] T.-H. Her, X. Ao, L. W. Casperson, The Univ. of North Carolina at Charlotte (United States)

757908 Shaping of dark beams in resonators with a bi-lens reflector [7579-08]

Y. N. Parkhomenko, B. Spektor, J. Shamir, Technion-Israel Institute of Technology (Israel)

757909 Propagation of 3D beams using a finite-difference algorithm: practical considerations [7579-09]

A. H. Paxton, Air Force Research Lab. (United States)

7579 OA Precision control of the transfer matrix method for transverse-mode fields of laser resonators [7579-10]

D. Ling, H. Wang, S. Zhang, Dongguan Univ. of Technology (China)

\section{MICRORESONATOR STRUCTURES AND MATERIALS}

7579 OC Engineering optical forces in waveguides and cavities based on optical response (Invited Paper) [7579-52]

P. T. Rakich, Sandia National Labs. (United States); Z. Wang, M. A. Popović, Massachusetts Institute of Technology (United States)

7579 OE Integration of chalcogenide and titanium-diffused lithium-niobate waveguides (Invited Paper) [7579-54]

M. E. Solmaz, C. K. Madsen, Texas A\&M Univ. (United States) 
$7579 \mathrm{OF}$ Generation of multikilowatt radially or azimuthally polarized $\mathrm{CO}_{2}$ laser beams by a triple-axicon optical resonator [7579-11]

M. Endo, Tokai Univ. (Japan)

7579 OG Tolerance analysis for stable laser resonators [7579-12]

M. Kuhn, LightTrans VirtualLab UG (Germany); F. Wyrowski, Friedrich-Schiller-Univ. Jena

(Germany); C. Hellmann, T. Schöning, LightTrans VirtualLab UG (Germany)

7579 ol Multi dither adaptive system based on Shack-Hartmann wavefront sensor [7579-04] J. Sheldakova, A. Kudryashov, V. Samarkin, A. Alexandrov, Moscow State Open Univ. (Russian Federation)

7579 0J Common cavity resonators for passive laser beam combining: effects of path length errors (Invited Paper) [7579-15]

J. R. Leger, M. Khajavikhan, C. Wan, Univ. of Minnesota (United States)

7579 OK A novel technology based on $\mathrm{CO}_{2}$ lasers for surface finishing and direct fiber fusion of beam delivery optics [7579-16]

G. Curatu, O. Ruiz, LightPath Technologies, Inc. (United States)

\section{BEAM SHAPING}

7579 OM Gaussian beam shaping based on multimode interference [7579-18]

X. Zhu, A. Schülzgen, H. Li, J. V. Moloney, N. Peyghambarian, College of Optical Sciences, The Univ. of Arizona (United States)

7579 ON Applying refractive beam shapers in creating spots of uniform intensity and various shapes [7579-19]

A. Laskin, MolTech GmbH (Germany); G. Williams, Univ. of Sheffield (United Kingdom);

A. Demidovich, ELETTRA-Sincrotrone (Italy)

757900 Overview: process-optimized beam transformers and their impact on high-power laser applications [7579-20]

O. Homburg, T. Mitra, L. Aschke, LIMO Lissotschenko Mikrooptik GmbH (Germany)

\section{BEAM CHARACTERIZATION AND DIAGNOSTICS}

7579 OR Complete description of optical fields propagating in passive LMA fibers regarding amplitude, relative phase delay, and polarization by means of optical correlation filters [7579-23]

D. Flamm, Friedrich-Schiller-Univ. Jena (Germany); S. Schröter, Institut für Photonische Technologien Jena (Germany); M. Duparré, Friedrich-Schiller-Univ. Jena (Germany)

7579 OT Spatial control and diagnostics of femtosecond pulses with programmable microoptics (Invited Paper) [7579-25]

R. Grunwald, M. Bock, Max Born Institute for Nonlinear Optics and Ultrafast Spectroscopy (Germany) 
7579 OU Fractional power in the bucket, beam quality and $M^{2}$ [7579-26]

S. Basu, Sparkle Optics Corp. (United States); L. M. Gutheinz, Boeing-SVS, Inc. (United States)

MICRORESONATORS: NONLINEAR APPLICATIONS, NOVEL DEVICES, AND SENSORS I

7579 OV Intracavity frequency conversion: from bow-ties to whispering galleries (Invited Paper) [7579-27]

I. Breunig, R. Sowade, J. Kießling, T. Beckmann, H. Linnenbank, D. Haertle, K. Buse, Univ. Bonn (Germany)

7579 OW Tuning of whispering gallery modes of polymeric micro-spheres and shells using external electric field [7579-28]

T. Ioppolo, U. Ayaz, M. V. Ötügen, Southern Methodist Univ. (United States)

7579 OY PDMS-based microfluidic lasers using whispering gallery modes for lab-on-a-chip applications [7579-30]

J. D. Suter, D. J. Howard, Univ. of Missouri, Columbia (United States); E. Hoppmann,

I. M. White, Univ. of Maryland, College Park (United States); X. Fan, Univ. of Missouri,

Columbia (United States)

MICRORESONATORS: CAVITY OPTOMECHANICS AND QED I

757916 Evanescent waves outside eccentric optical cavities [7579-38]

S. C. Creagh, M. White, The Univ. of Nottingham (United Kingdom)

MICRORESONATORS: CAVITY OPTOMECHANICS AND QED II

757918 Hybrid approaches toward single emitter coupling to optical microresonators (Invited Paper) [7579-40]

M. Barth, M. Gregor, R. Henze, T. Schröder, Humboldt-Univ. zu Berlin (Germany); N. Nüsse,

B. Löchel, Helmholtz-Zentrum Berlin für Materialien und Energie GmbH (Germany);

O. Benson, Humboldt-Univ. zu Berlin (Germany)

757919 Slow light rotation sensors and gyroscopes (Invited Paper) [7579-41]

J. Schever, Tel Aviv Univ. (Israel)

7579 1A Three-mode opto-acoustic interactions in optical cavities: introducing the three-mode opto-acoustic parametric amplifier (Invited Paper) [7579-42]

F. A. Torres, D. G. Blair, L. Ju, C. Zhao, H. Miao, The Univ. of Western Australia (Australia)

MICRORESONATORS: NONLINEAR APPLICATIONS, NOVEL DEVICES, AND SENSORS II

7579 1B High-Q optical resonators: characterization and application to stabilization of lasers and high spectral purity microwave oscillators (Invited Paper) [7579-43]

O. Llopis, P. H. Merrer, A. Bouchier, K. Saleh, LAAS-CNRS (France) and Univ. de Toulouse (France); G. Cibiel, CNES (France) 
7579 1C Ultra-narrowband fiber Bragg gratings for laser linewidth reduction and RF filtering (Invited Paper) [7579-44]

M. Poulin, Y. Painchaud, M. Aubé, S. Ayotte, C. Latrasse, G. Brochu, F. Pelletier, M. Morin, M. Guy, J.-F. Cliche, TeraXion Inc. (Canada)

7579 1D Single cavity filters on end-faces of optical fibers [7579-45]

S. Meister, D. Schweda, M. Dziedzina, A. Al-Saadi, B. A. Franke, C. Scharfenorth, Technische Univ. Berlin (Germany); B. Grimm, D. Dufft, S. K. Schrader, Technische Fachhochschule Wildau (Germany); H. J. Eichler, Technische Univ. Berlin (Germany)

\section{LASE PLENARY SESSION}

7579 IF Ultrafast fiber laser technology: status and prospects (Plenary Paper) [7579-102]

A. Tünnermann, J. Limpert, Friedrich-Schiller-Univ. Jena (Germany) and Fraunhofer Institute for Applied Optics and Precision Engineering (Germany)

\section{POSTER SESSION}

7579 IG Optical-axis perturbation in triaxial ring resonators II: induced by spherical mirror's axial displacement [7579-47]

X. Long, J. Yuan, National Univ. of Defense Technology (China)

757911 Spectra, coherence, and polarization of diffracted electromagnetic Gaussian Schell-model beams [7579-49]

L. Pan, Luoyang Normal Univ. (China); C. Ding, Sichuan Univ. (China); Z. Zhao, Luoyang Normal Univ. (China)

$75791 \mathrm{~J}$ Temperature-dependent random lasing from GaAs powders [7579-50]

T. Nakamura, T. Takahashi, S. Adachi, Gunma Univ. (Japan)

Author Index 


\title{
Conference Committee
}

\author{
Symposium Chairs
}

Donald J. Harter, IMRA America, Inc. (United States)

Peter R. Herman, University of Toronto (Canada)

Symposium Cochairs

Alberto Piqué, Naval Research Laboratory (United States)

Friedhelm Dorsch, TRUMPF Photonics (United States)

Program Track Chair

Gregory J. Quarles, VLOC (United States)

Conference Chairs

Alexis V. Kudryashov, Moscow State Open University (Russian Federation)

Alan H. Paxton, Air Force Research Laboratory (United States)

Vladimir S. Ilchenko, OEwaves, Inc. (United States)

Conference Cochair

Lutz Aschke, LIMO Lissotschenko Mikrooptik GmbH (Germany)

Program Committee

Jean-Claude M. Diels, The University of New Mexico (United States)

Hans-Joachim Eichler, Technische Universität Berlin (Germany)

Andrew Forbes, Council for Scientific and Industrial Research

(South Africa)

Pierre Galarneau, Institut National d'Optique (Canada)

Thomas Graf, Universität Stuttgart (Germany)

James R. Leger, University of Minnesota (United States)

Andrey B. Matsko, OEwaves, Inc. (United States)

Shayan Mookherjea, University of California, San Diego (United States)

Steve A. Pappert, Defense Advanced Research Projects Agency

(United States)

Michael J. Scaggs, Haas Laser Technologies, Inc. (United States) 
Session Chairs

$1 \quad$ Adaptive Optics

Alexis V. Kudryashov, Moscow State Open University

(Russian Federation)

Alan H. Paxton, Air Force Research Laboratory (United States)

2 Adaptive Optics and Laser Simulation

Vladimir S. Ilchenko, OEwaves, Inc. (United States)

3 Microresonator Structures and Materials

Greeshma Gupta, University of California, San Diego (United States)

$4 \quad$ Laser Beam Control and Applications in Manufacturing

Shayan Mookherjea, University of California, San Diego (United States)

5 Beam Shaping

Alan H. Paxton, Air Force Research Laboratory (United States)

6 Beam Characterization and Diagnostics

James R. Leger, University of Minnesota (United States)

7 Microresonators: Nonlinear Applications, Novel Devices, and Sensors I

Stefan Meister, Technische Universität Berlin (Germany)

8 Microresonators: Photonics Applications

Michael J. Scaggs, Haas Laser Technologies, Inc. (United States)

9 Microresonators: Cavity Optomechanics and QED I

Andrey B. Matsko, OEwaves, Inc. (United States)

10 Microresonators: Cavity Optomechanics and QED ॥

Lutz Aschke, LIMO Lissotschenko Mikrooptik GmbH (Germany)

11 Microresonators: Nonlinear Applications, Novel Devices, and Sensors II

Ryan P. Lu, Space and Naval Warfare Systems Command (United States)

LASE Plenary Session

Donald J. Harter, IMRA America, Inc. (United States)

Peter R. Herman, University of Toronto (Canada) 